\title{
Superstring field theory in the
}

\section{democratic picture}

\author{
Michael Kroyter ${ }^{1,2}$ \\ ${ }^{1}$ Center for Theoretical Physics, Massachusetts Institute of Technology, \\ Cambridge, MA 02139, USA \\ ${ }^{2}$ School of Physics and Astronomy, The Raymond and Beverly Sackler \\ Faculty of Exact Sciences, Tel Aviv University, Ramat Aviv 69978, Israel \\ mikroyt@tau.ac.il
}

\begin{abstract}
We present a new open superstring field theory, whose string fields carry an arbitrary picture number and reside in the large Hilbert space. The redundancy related to picture number is resolved by treating picture changing as a gauge transformation. A mid-point insertion is imperative for this formalism. We find that this mid-point insertion must include all multi-picture-changing operators. It is also proven that this insertion as well as all the multi-picture-changing operators are zero weight conformal primaries. This new theory solves the problems with the Ramond sector shared by other Ramond-Neveu-Schwarz (RNS) string field theories, while naturally unifying the Neveu-Schwarz (NS) and Ramond string fields. When partially gauge fixed, it reduces in the NS sector to the modified cubic superstring field theory. Hence, it shares all the good properties of this theory, e.g., it has analytical vacuum and marginal deformation solutions. Treating the redundant gauge symmetry using the Batalin-Vilkovisky (BV) formalism is straightforward and results in a cubic action with a single string field, whose quantum numbers are
\end{abstract}

e-print archive: http://lanl.arXiv.org/abs/0911.2962v2 
unconstrained. The generalization to an arbitrary brane system is simple and includes the standard Chan-Paton factors and the most general string field consistent with the brane system.

\section{Contents}

1 Introduction $\quad 742$

$\begin{array}{lll}2 & \text { Cubic superstring field theory } & \mathbf{7 4 4}\end{array}$

3 Cohomology in the large Hilbert space $\quad 747$

4 Constructing the theory $\quad 756$

4.1 Constructing the form of the theory 756

$\begin{array}{lll}4.2 & \text { Constructing the mid-point insertion } & 757\end{array}$

5 The Ramond sector and general D-brane systems $\quad 765$

$6 \quad$ Field-antifield formulation and gauge fixing 766

$\begin{array}{lll}7 & \text { Supersymmetry } & 769\end{array}$

8 Conclusions $\quad \mathbf{7 7 4}$

$\begin{array}{lr}\text { Acknowledgments } & 777\end{array}$

$\begin{array}{lr}\text { References } & 777\end{array}$

\section{Introduction}

The existence of picture number is a central characteristic of the RNS formalism of superstring theory [1]. Picture number complicates the construction of RNS string field theories. ${ }^{1}$ The fact that the pictures of the NS fields are integer, while those of the Ramond fields are half-integer implies that one should put either a relative picture-changing operator between the two sectors, as is done in the modified theory [5], or use two (or more) Ramond

\footnotetext{
${ }^{1}$ Various steps towards the construction of superstring field theory can be found in [2-17]. A review of recent progress in string field theory, whose Section 8 includes an introduction to superstring field theory is [18].
} 
fields with a constraint, as in the non-polynomial version of the theory [12]. Both these resolutions are problematic. The former suffers from collisions of picture- changing operators $[4,16],{ }^{2}$ while the constraint of the latter cannot be derived from a covariant action. We review the current status of RNS superstring field theories, focusing on the cubic theories, in Section 2.

In this work we propose a new open RNS string field theory, which does not suffer from these problems. Our starting point in defining this action relies on two observations made in [20]. The first is that the cohomology of $Q$ at any given picture and ghost numbers in the small Hilbert space is the same as the cohomology of $Q-\eta_{0}$ at the same picture and ghost numbers in the large Hilbert space. The second observation is that relaxing the constraint of a given picture number does not change the cohomology, unlike the case of $Q$ in the small Hilbert space, where considering two picture numbers at once results in a doubling of the cohomology. We explain these issues in Section 3.

The theory itself is introduced in Section 4. The action is cubic and is defined in the large Hilbert space,

$$
S=-\oint \mathcal{O}\left(\frac{1}{2} \Psi \tilde{Q} \Psi+\frac{1}{3} \Psi^{3}\right) .
$$

Here, $\Psi$ is the string field and we abbreviated

$$
\tilde{Q} \equiv Q-\eta_{0} .
$$

The string fields are multiplied, as always, using Witten's star product [2], which we keep implicit. The integration symbol appearing in (1.1) is used throughout this paper to represent the conformal field theory (CFT) expectation value in the large Hilbert space, while the standard integration symbol represents the expectation value in the small Hilbert space. The mid-point insertion $\mathcal{O}$ is defined using a superposition of all multi-picture-changing operators. We introduce these operators and discuss their properties. In particular, it is proven that they can always be represented by primary fields.

Another novel property of the action (1.1), which we present in Section 5, is that the NS and Ramond sector fields are simply unified into a single string field

$$
\Psi \rightarrow \Psi+\alpha .
$$

Now, $\Psi$ is the NS string field, which is formed by a linear combination of string fields with arbitrary integer picture number and $\alpha$ is the Ramond

\footnotetext{
${ }^{2}$ Witten's theory also does not seem to support some desired classical solutions [19].
} 
string field, which is formed by a linear combination of half-integer picture numbers. Expanding the action (1.1) in terms of the new $\Psi$ and $\alpha$ gives

$$
S=-\oint \mathcal{O}\left(\frac{1}{2} \Psi \tilde{Q} \Psi+\frac{1}{3} \Psi^{3}+\frac{1}{2} \alpha \tilde{Q} \alpha+\Psi \alpha^{2}\right) .
$$

This is very similar in form to the usual cubic RNS superstring field theory action [5]. The most striking difference being the fact that the same insertion $\mathcal{O}$ multiplies all terms in the action. The consistent inclusion of the Ramond sector enables the covariant description of all sectors of general D-brane systems in the new formalism.

The field-antifield (BV) formulation of the theory is presented in Section 6 , where we also comment on gauge fixing. The supersymmetry properties of the theory are discussed in Section 7. Conclusions and some open problems are presented in Section 8.

Note added: While this work was nearing completion, I learned of the work [21], which has some similarities to our construction. It would be interesting to understand the interrelation, if any, between the two theories.

\section{Cubic superstring field theory}

In this section we recall the construction of the existing cubic RNS string field theories and their problems.

The first proposal for such a theory was presented by Witten [3], following his bosonic theory [2]. While the structure in the two cases is almost the same, there was one essential new feature that had to be addressed, namely the picture number. Since in the small Hilbert space, where the theory was constructed, CFT expectation values are non-zero for picture number -2 , it seemed quite sensible to define the NS string field to carry the "natural" -1 picture, in order to obtain a standard form for the kinetic term in the action.

The interaction term, on the other hand, had to be appended with a +1 picture number, in order to allow for a non-trivial result. This was achieved by an explicit insertion of the picture-changing operator $X$ in the action. The only consistent way of inserting the picture-changing operator was as a mid-point insertion. Any other choice would have destroyed the associativity of the star product and the gauge invariance of the action. However, it was soon realized that the facts that the mid-point is invariant under the star product and that the operator product expansion (OPE) of $X$ with itself is singular imply the emergence of singularities that ruin the consistency of the theory [4]. 
The resolution of $[5,7]$ (henceforth, "the modified theory") was to change the picture number of the NS string field to zero and insert an overall factor of $Y_{-2}$ in front of the action. The action of the NS sector of this construction is given by

$$
S_{\mathrm{NS}}=-\int Y_{-2}\left(\frac{1}{2} \Psi Q \Psi+\frac{1}{3} \Psi^{3}\right) .
$$

Now, the gauge transformation does not contain any picture-changing operators and in perturbation theory the factors of $Y_{-2}$ come (at least for trees) next to factors of $\left(Y_{-2}\right)^{-1}$ from the propagator. Therefore, singularities do not emerge.

While this solved the problem with picture-changing collisions, other criticism on this theory remained. A common claim was that the equation of motion derived from the action is not the one needed, due to the non-trivial kernel of $Y_{-2}$,

$$
Y_{-2}\left(Q \Psi+\Psi^{2}\right)=0 \stackrel{?}{\Longleftrightarrow} Q \Psi+\Psi^{2}=0 .
$$

In fact, not only the kernel of $Y_{-2}$, but also the space of operators whose OPE with $Y_{-2}$ is singular, is potentially problematic. However, these problems may arise only for string fields having these types of operators as local insertions at the string mid-point. String fields of this sort suffer from problems regardless of the existence of the mid-point insertion in the action and (at least) most of them should be discarded from the construction of the space of string fields. ${ }^{3}$ While the construction of a space of string fields is generally lacking, it does not seem that it would be more complicated in the case of the modified theory. We therefore believe that this issue does not form a ground for discarding the theory. Another issue of this theory is the abundance of possible $Y_{-2}$ insertions. However, it was shown in [17] that theories based on different choices of $Y_{-2}$ 's are classically equivalent. Hence, this is also not much of an obstruction to the theory. Moreover, cubic superstring field theory is very successful in many ways. In particular, analytical solutions describing the tachyon vacuum and marginal deformations are known in the theory [22-24].

\footnotetext{
${ }^{3}$ See, for example, section 2 of [15] for further related discussion. Also note that a cubic theory, which avoids insertions of operators with a non-trivial kernel, exists and it seems that it is classically equivalent to the modified theory $[14,15]$. However, this formalism does not solve the problems with the Ramond sector. Moreover, the space of operators whose OPE with the insertion of this theory is singular, is non-trivial. Thus, even if one insists, in contrast to what we suggest here, that mid-point insertions should be allowed, this theory is still in no way better than the modified one.
} 
There is, however, one serious problem with this formalism related to the inclusion of the Ramond sector. The Ramond sector carries half-integer pictures. Hence, it is impossible (with a Ramond field of a well-defined picture) to write an interacting action with a common $Y_{-2}$ factor. The proposal of [5] was to generalize the action (2.1) to

$$
S=-\int\left(Y_{-2}\left(\frac{1}{2} \Psi Q \Psi+\frac{1}{3} \Psi^{3}\right)+Y\left(\frac{1}{2} \alpha Q \alpha+\Psi \alpha^{2}\right)\right),
$$

where $\alpha$ is the Ramond field.

That this formulation is problematic can be observed already when deriving the equations of motion from the action (2.3). These take the form (after acting on them with picture-changing operators)

$$
\begin{aligned}
Q \Psi+\Psi^{2}+X \alpha^{2} & =0 \\
Q \alpha+[\Psi, \alpha] & =0 .
\end{aligned}
$$

The first of these equations implies that mid-point insertions are to be allowed for at least one of the string fields $\Psi$ and $\alpha$, since otherwise the Ramond sector becomes trivial. It is not clear what sort of an operator could have been inserted on $\alpha$, in order to produce a $Y$ (that is needed to cancel the $X$ ) on $\alpha^{2}$. Hence, we assume that the insertion is on $\Psi$. We cannot assign $X$ to $\Psi$, since then the term $\Psi^{2}$ would be singular. Hence, we decompose $\Psi$ as

$$
\Psi=\Psi_{0}+\xi \Psi_{1},
$$

with $\Psi_{0}$ being an odd component and $\Psi_{1}$ being an even one [16]. This still does not solve the problem, since singular terms of the form $\xi X$ from the $\Psi Q \Psi$ term appear now in the action. One may claim that the $X$ should be cancelled against "half the $Y_{-2}$ " before it is multiplied by the $\xi$. This means, however, that all string fields in the theory should be regularized somehow, already at the classical level. In fact, the theory itself should be regularized.

A similar observation can be made by considering the gauge transformation of this theory:

$$
\begin{aligned}
\delta \Psi & =Q \Lambda+[\Psi, \Lambda]+X[\alpha, \chi], \\
\delta \alpha & =Q \chi+[\alpha, \Lambda]+[\Psi, \chi],
\end{aligned}
$$

where $\Lambda$ is the NS gauge string field and $\chi$ is the Ramond gauge string field, both of which are even string fields. While the second equation is benign, the first one explicitly shows that an $X$ mid-point insertion should be allowed 
for $\Psi$, as it is not clear how one could avoid it without moving the problem to $\alpha$. In fact, when iterated, (2.6) inevitably leads to singularities due to collisions of $X[16]$.

It might be possible to regularize the theory by moving the local insertions away from the mid-point. However, such a regularization would break the associativity of the star product and the gauge invariance of the theory. It is not clear whether those could be restored in the limit in which the regularization is removed, nor is it clear whether the singularities can be avoided in this limit. Another option would be to look for an insertionfree formulation. Such a formulation exists, namely the non-polynomial theory $[8,12]$. Here, however, one faces another problem. The theory is defined using two constrained Ramond fields. If one insists on a covariant formulation of the theory, the constraint cannot be derived from an action.

One might try to use two Ramond fields in the description of the cubic theory, in analogy with the situation in the non-polynomial theory. While the resulting theory has some nice properties, a constraint relating the two Ramond fields should be introduced [16]. Nonetheless, it is not understood how to derive a proper constraint from an action without introducing explicit mid-point insertions. Thus, the problems described above persist. We conclude that a more fundamental revision of the theory is needed. We derive a cubic theory with the desired properties in Section 4.

\section{Cohomology in the large Hilbert space}

In this section we review the various representations of vertex operators in the RNS formalism. Vertex operators are important to us, since string fields are their off-shell generalizations. Different choices of representations of the vertex operators naturally lead to different string field theory formulations. While in their most familiar representation, the RNS vertex operators live in the small Hilbert space, some other useful representations use the large Hilbert space.

The large Hilbert space consists of two copies of the small Hilbert space, with one of the copies being multiplied by $\xi_{0}$ [1]. Within the copy without the $\xi_{0}$ insertion, i.e., within the small Hilbert space, the on-shell condition for a vertex operator $V$ gets the form

$$
Q V=0 .
$$

The requirement of being in the small Hilbert space is enforced by

$$
\eta_{0} V=0 .
$$


To these relations one has to add the identification of states that differ by an exact element

$$
V \approx V+Q \Lambda,
$$

where $\Lambda$ also obeys (3.2). This is the way that the standard cohomology problem is formulated in the large Hilbert space. The cubic theories described in Section 2 are off-shell extensions of this representation of the vertex operators.

If one wishes to consider the second copy of the small Hilbert space, i.e., the one with the $\xi_{0}$ insertion, the on-shell condition for a vertex operator $V$ can be written as

$$
Q \eta_{0} V=0 .
$$

Here $\eta_{0}$ removes the $\xi_{0}$ insertion and the remaining equation is the same as (3.1). Two solutions should be considered equivalent if they differ by a term of the form

$$
\delta V=\xi_{0} Q \eta_{0} \Lambda .
$$

This relation mimics (3.3) for the $\xi_{0}$ copy of the small Hilbert space. Hence, (3.4) and (3.5) correctly define the equivalence classes in this space, despite the fact that they do not look like a cohomology problem. The expression (3.4) can also be used for the whole large Hilbert space. To that end we have to append to the equivalence relation (3.5) the equivalence to zero of the small Hilbert space. Changing the basis of equivalence generators this can be written as

$$
\delta V=Q \Lambda_{Q}+\eta_{0} \Lambda_{\eta} .
$$

There is a natural correspondence between $\xi_{0}$-based states at ghost and picture numbers $g$ and $p$ and small Hilbert space states at ghost number $g+1$ and picture number $p-1$. The change of quantum numbers comes from using $\eta_{0}$ and $\xi_{0}$ as the canonical isomorphism mappings. Hence, NS string fields in the "natural," $p=-1, g=1$ picture, correspond to $p=g=0$ string fields that extend the vertex operators represented by (3.4) and (3.6). While the former case leads to Witten's theory [3], which suffers from divergences, the later naturally leads to the non-polynomial theory of Berkovits [8], in which these problems do not arise.

One might wish at this stage to consider also the cohomology of $Q$ in the large Hilbert space. Another possibility would be to consider the cohomology 
of $\eta_{0}$, since using ${ }^{4}$

$$
\left[Q, \eta_{0}\right]=0
$$

one realizes that (3.4) and (3.6) are exactly symmetric upon interchanging $Q$ and $\eta_{0}$. However, in the large Hilbert space the cohomology of both operators is trivial, as we turn now to show while further illustrating the symmetry between both operators. ${ }^{5} \quad$ For an arbitrary derivation $d$, the triviality of its cohomology can most easily be shown if a state $A$ exists such that $d A=\mathbf{1}$, where $\mathbf{1}$ is the identity element of the algebra on which $d$ acts. ${ }^{6}$ The proof is then straightforward. Let $V$ be a closed state, i.e., $d V=0$, then $V$ is exact. Specifically, $V=d(A V)$, since

$$
d(A V)=(d A) V+(-)^{(d)(A)} A d V=\mathbf{1} V+0=V,
$$

where $(d)$ and $(A)$ in the exponent stand for the parities of the derivation and the state $A$. Such a state exists for $\eta_{0}$. In fact, a family of such states exists, namely $\xi(z)$. It is less trivial to find it, but a similar family exists for $Q[29]$ namely $^{7}$

$$
P(z)=-c \xi \partial \xi \mathrm{e}^{-2 \phi}(z)
$$

Hence, we write

$$
\begin{aligned}
& Q P(z)=1, \\
& \eta_{0} \xi(z)=1,
\end{aligned}
$$

\footnotetext{
${ }^{4}$ Here and elsewhere, $[A, B]$ stands for the graded commutator, e.g., $\left[Q, \eta_{0}\right] \equiv Q \eta_{0}+$ $\eta_{0} Q$.

${ }^{5}$ The symmetry between $Q$ and $\eta_{0}$ was noted already by Berkovits and Vafa [25]. In the $N=4$ language $J_{B}$ and $\eta$ are the currents $G^{+}$and $\tilde{G}^{+}$, respectively. Also, note that one can completely exchange the roles of $Q$ and $\eta_{0}$ and define a "dual small Hilbert space," by considering the $Q$-closed subspace of the large Hilbert space and studying the cohomology of $\eta_{0}$ in this space. This cohomology is the same as that of $Q$ in the "ordinary small Hilbert space," since both ( $Q$ 's cohomology in one space and $\eta_{0}$ 's in the other) are relative cohomologies that are defined in the same way in the large Hilbert space: $\eta_{0} V=Q V=0$, $V \approx V+Q \eta_{0} \Lambda$.

${ }^{6}$ Such a state is called a "contracting homotopy." In the context of string field theory this structure was used for proving that the cohomology around Schnabl's solution [26] is trivial [27] (see also $[22,24,28]$ ).

${ }^{7}$ It is easy to read from $P$ the part of $Q$, which has $\phi$-momentum two. However, the complete $Q$ is related to this part by a similarity transformation [30] and the generator of this transformation leaves $P$ invariant.
} 
where 1 here stands for $\mathbf{1}(z)$, the insertion of unity at $z$, which does not change the state on which it acts and is of course $z$-independent. This completes the proof of the triviality of $Q$ and $\eta_{0}$.

It is also interesting to consider

$$
\begin{aligned}
& X(z) \equiv Q \xi(z)=\left(c \partial \xi+\mathrm{e}^{\phi} G_{m}+\mathrm{e}^{2 \phi} b \partial \eta+\partial\left(\mathrm{e}^{2 \phi} b \eta\right)\right)(z), \\
& Y(z) \equiv \eta_{0} P(z)=\left(c \partial \xi \mathrm{e}^{-2 \phi}\right)(z) .
\end{aligned}
$$

Here, $G_{m}$ is the superconformal matter generator, which in flat background takes the form ${ }^{8}$

$$
G_{\mathrm{m}}=\mathrm{i} \psi_{\mu} \partial X^{\mu} .
$$

Expression (3.11a) is, however, universal and holds regardless of the existence of a specific background.

These definitions imply that all the quantum numbers of $X$ and $Y$ are trivial except for their picture numbers, which are 1 and -1 , respectively. In particular, they are zero weight conformal primaries. These operators are also $Q$-closed. For $X$ it follows from the fact that it is exact (in the large Hilbert space), while for $Y$ it follows from the (graded) Jacobi identity and the relations (3.7) and (3.10a). Similarly, these operators are closed with respect to $\eta_{0}$. All in all we can write

$$
Q X=Q Y=\eta_{0} X=\eta_{0} Y=0 .
$$

It is also possible to write $Y$ explicitly as an exact state in the large Hilbert space

$$
Y=Q \mathcal{Y} .
$$

This statement is in a sense trivial, since we already proved that it is closed and any closed state can be written in the large Hilbert space as an exact state using $P$. However, $P$ has a singular OPE with $Y$, which complicates the explicit construction. The most straightforward resolution of the OPE singularities would have been the replacement of the operator product by a normal ordered product. This strategy does not work, since it leads to a vanishing result, due to the zeros at the $b c$ and $\xi \eta$ sectors. We can remedy that by defining $\mathcal{Y}$ as the leading regular term in the OPE,

$$
\mathcal{Y}_{0}(w) \equiv \oint \frac{d z}{2 \pi \mathrm{i}} \frac{P(z) Y(w)}{z-w} .
$$

\footnotetext{
${ }^{8}$ We use the same conventions as in [18].
} 
This is almost what we want. This operator is local and obeys (3.14). However, it has some non-trivial quantum numbers other than the needed ghost and picture numbers, meaning that, while it carries zero conformal weight, it is not a primary conformal field. Since this property will turn out to be of importance to us, we would like to suggest a conformal primary candidate for $\mathcal{Y}$,

$$
\mathcal{Y}=\frac{1}{5} c \xi \partial \xi \mathrm{e}^{-3 \phi} G_{m}-\xi \mathrm{e}^{-2 \phi} .
$$

Similarly, $X$ can be written, in the large Hilbert space, as a local $\eta_{0}$ exact state.

From the discussion above it follows that

$$
P=\xi Y, \quad \xi=P X .
$$

The last two equalities in (3.13) imply that $X$ and $Y$ reside in the small Hilbert space. We can now conclude that these operators are "picturechanging operators," namely that they define homomorphisms between the cohomologies (in the small Hilbert space) of $Q$ at picture numbers $p$ and $p \pm 1$. They are also each others inverse in the sense of the OPE,

$$
X(z) Y(0) \sim 1,
$$

as is implied by (3.17). Nevertheless, as already stated, these operators suffer from singularities in their OPEs with themselves:

$$
\begin{array}{ll}
X(z) X(0) \sim \frac{(\cdots)}{z^{2}}, & \xi(z) X(0) \sim \frac{(\cdots)}{z^{2}}, \\
Y(z) Y(0) \sim \frac{(\cdots)}{z^{2}}, & P(z) Y(0) \sim \frac{(\cdots)}{z^{2}} .
\end{array}
$$

If locality is not important, one can plug several operators at different values of $z$ and obtain singularity-free multi-picture-changing operators in this way. Otherwise, the singular parts of the OPEs can be simply removed, since they correspond to $Q$-exact terms.

With the understanding of picture-changing operators one can define more ways for representing the cohomology problem in the large Hilbert space [20]. In these new representations, the physical space could reside in the small Hilbert space and not in its $\xi_{0}$ copy as before. Hence, the ghost and picture numbers are the same as for the $Q$ cohomology. The cohomology operator that one should use is $\tilde{Q}$ of (1.2). 
Let $V$ be a closed state with respect to $\tilde{Q}$, i.e.,

$$
\tilde{Q} V=0,
$$

and let it have a given picture number $p$. Then, since $Q V$ and $\eta_{0} V$ have different picture numbers, (3.20) implies that

$$
Q V=\eta_{0} V=0,
$$

i.e., $V$ lives in the small Hilbert space and is closed. Suppose we add to $V$ a term of the form $\tilde{Q} \Lambda$, subject to the constraint that the result still has picture number $p$. Such a $\Lambda$ can be decomposed to picture numbers $p$ and $p+1$ plus a term $\Lambda_{\text {triv }}$ obeying

$$
Q \Lambda_{\text {triv }}=\eta_{0} \Lambda_{\text {triv }}=0 .
$$

Such a term does not contribute to $\delta V$ and so can be discarded. The requirement that $\delta V$ has picture number $p$ implies

$$
Q \Lambda_{p+1}=0, \quad \eta_{0} \Lambda_{p}=0
$$

The triviality of $Q$ and $\eta_{0}$ in the large Hilbert space then implies

$$
\Lambda_{p+1}=Q \Lambda_{p+1}^{Q}, \quad \Lambda_{p}=\eta_{0} \Lambda_{p+1}^{\eta} .
$$

All in all we can write

$$
\delta V=\tilde{Q} \Lambda=\left(Q-\eta_{0}\right)\left(Q \Lambda_{p+1}^{Q}+\eta_{0} \Lambda_{p+1}^{\eta}\right)=Q \eta_{0}\left(\Lambda_{p+1}^{Q}+\Lambda_{p+1}^{\eta}\right),
$$

that is, two states are identified if they differ by a state, which is $Q$ exact in the small Hilbert space. Given such a variation,

$$
\delta V=Q \Lambda,
$$

with $\Lambda$ in the small Hilbert space, one can choose

$$
\Lambda_{p}=\Lambda, \quad \Lambda_{p+1}=0,
$$

and get this variation as a variation by a $\tilde{Q}$ exact term in the large Hilbert space. Hence, the cohomology of $Q$ in the small Hilbert space is the same as that of $\tilde{Q}$ in the large Hilbert space at the same picture and ghost numbers, as stated. 
Unlike the case of the usual cohomology problem, the cohomology does not change upon relaxing the fixed picture condition. Assume that the state $V$ carries a picture number bounded between $p_{\min }$ and $p_{\max }$. We write

$$
V=\sum_{p=p_{\min }}^{p_{\max }} V_{p} .
$$

Consider the gauge transformation generated by ${ }^{9}$

$$
\Lambda=\xi(z) V_{p_{\min }}
$$

It induces the variation

$$
\delta V=\left(Q-\eta_{0}\right) \xi(z) V_{p_{\min }}=(X-\xi Q) V_{p_{\min }}-V_{p_{\min }}+\xi \eta_{0} V_{p_{\min }} .
$$

The first term has picture number $p_{\min }+1$, the second term eliminates the original $V_{p_{\min }}$, while the last term drops out, since it is the lowest picture component of (3.20). Thus, this gauge transformation removes the lowest picture of the state. If one considers instead the gauge transformation generated by

$$
\Lambda=-P(z) V_{p_{\max }},
$$

one removes the highest picture component, since now

$$
\delta V=\left(Y-P \eta_{0}\right) V_{p_{\max }}-V_{p_{\max }}
$$

Using these transformations, one can reduce the picture number range of any state to a single arbitrary picture number

$$
p_{\min } \leq p \leq p_{\max }
$$

For this case, we have already shown that the $\tilde{Q}$ cohomology is equivalent to the $Q$ cohomology. Moreover, the equivalence to the $Q$ cohomology is independent of the choice of the final $p$. To show that, consider the one before last stage in the sequence of gauge transformations, where two non-trivial

\footnotetext{
${ }^{9}$ Having string field theory in mind, we refer to a change of the representative of a given cohomology as a gauge transformation.
} 
picture numbers $p$ and $p+1$ are left. Using the gauge transformation (3.29) or (3.31), we can get to either of

$$
\begin{aligned}
V_{p}+V_{p+1} & \rightarrow V_{p}+Y V_{p+1}-P \eta_{0} V_{p+1} \\
V_{p}+V_{p+1} & \rightarrow X V_{p}+V_{p+1}-\xi Q V_{p} .
\end{aligned}
$$

For the first two terms on the r.h.s of the two equations, it is obvious that they are picture changed versions of the same state. For the last term it follows from (3.17) and

$$
Q V_{p}-\eta_{0} V_{p+1}=0
$$

which is the $p$-picture component of (3.20) for this case. We can now conclude that the cohomology of $\tilde{Q}$ over the space of states with picture number, which is arbitrarily bounded from both sides, is canonically isomorphic to that of $Q$ at any fixed picture number.

Assume now that the picture number is unbounded. By indefinitely repeating the procedure defined above, one can send the picture to arbitrarily high or low values. Hence, the state component at any given picture eventually will be zero. Explicitly, consider, the multi-picture-changing operators $X_{n}$ and $Y_{-n}$, for $n \geq 0$. These operators are the regularized versions of the powers of $X$ and $Y$, which are otherwise divergent and will be properly defined in the next section. In particular,

$$
X_{1}=X, \quad Y_{-1}=Y, \quad X_{0}=Y_{0}=1 .
$$

Among other properties, theses operators satisfy the relations

$$
\begin{aligned}
Q X_{n} & =Q Y_{-n}=\eta_{0} X_{n}=\eta_{0} Y_{-n}=0, \\
X Y_{-n} & \sim Y_{-(n-1)}, \quad Y X_{n} \sim X_{n-1} .
\end{aligned}
$$

Using these operators we can show that any $\tilde{Q}$-closed state is also exact, since the following contracting homotopy operators exist:

$$
\Lambda_{-}=\xi \sum_{n=1}^{\infty} Y_{-n}, \quad \Lambda_{+}=-P \sum_{n=1}^{\infty} X_{n} .
$$

One might mistakenly conclude that the cohomology of $\tilde{Q}$ over the space of states with unbounded picture number is trivial. However, there is always more than one way to define spaces using infinite sums of objects living in some constituent spaces. In the case at hand, we assumed that 
convergence in the all-picture space is defined "point-wise," i.e., as the union of the limits at every picture. There are certainly other ways to define limits. Let us use an analogy with the following sequence of vectors, $(1,0, \ldots),(1,1,0, \ldots),(1,1,1,0, \ldots), \ldots$ The point-wise limit of this sequence exists and equals $(1,1,1, \ldots)$. However, its limit in the $L_{1}$ norm, for instance, does not exist, since the norm of the "would-be limit" diverges.

One could look for the analogy of the above for the case of vertex operators. Any physical vertex operator can be represented in all possible pictures. Moreover, there would also be many exact terms that could be added to it. We can construct a $\mathbb{Z}$-sequence for any infinite sum of representatives of the physical vertex operator in the following way. We identify the location along the vector with the picture number. We choose a representation for the vertex operator at some given picture number and associate it with the vector $(\ldots, 0,0,1,0,0, \ldots)$. Next, we identify all the exact states with the zero vector and let the operators $X$ and $Y$ shift the vectors to the left and to the right. These rules establish a unique assignment of vectors. We now want to constrain the space of vectors by imposing some norm on it. The $L_{n}$ norms are probably not what we are after, since it is natural to expect that the norm commutes with the operations $X$ and $Y$. Instead, consider the absolute value of the sum of all entries, provided it is well defined regardless of the summation order. This is only a seminorm, since there are many elements whose "norm" is zero, i.e., all the exact states and all the states that are represented by vectors with entries that sum up to zero. Nonetheless, the semi-norm is enough for defining the space that we need. One can, as usual, divide this space by the trivial space and obtain a genuine norm. The resulting normed space would be one dimensional and it would correspond to the cohomology problem of this vertex.

We do not know how to generalize this construction to cover the whole space of string fields, since we do not know how should the relative normalization of different physical vertices work, nor do we know what to do with non-closed, i.e., off shell states. This is, however, exactly the usual problem with the definition of the space of string fields, which cannot be accomplished due to a lack of a natural norm. We conclude that in this respect the democratic theory is not better, but also not worse than any other string field theory. We would also like to point out that the correct definition of the space of string fields would probably differ from the one obtained using the semi-norm presented above, even when restricted to a given physical vertex, due to non-linearities. Specifically, we see below that the gauge transformation associated with picture changing should be modified in the case of an interacting theory. 
We saw that depending on the exact definition of the space of string fields with unbounded picture number, $\tilde{Q}$ has a trivial cohomology, or the same cohomology as for the bounded case. Presumably, it might also be possible to get other results for its cohomology. However, since we do not have a complete definition for the space of string field, we follow the "standard" practice in string field theoretical research and ignore this problem, implicitly assuming that this space is somehow defined in a proper way.

\section{Constructing the theory}

Here, we want to define an RNS string field theory, which generalizes the $\tilde{Q}$-description of vertex operators. It turns out that a single or a bounded range of picture numbers are inadequate choices. Hence, the theory will generalize the case where all picture numbers are allowed. We say that the theory is defined in the "democratic picture," since within this construction, all string fields, regardless of picture numbers, have equal opportunity to influence the physics. ${ }^{10}$ The construction of the theory is almost straightforward. The only subtlety is that a non-trivial insertion is required. We start this section by deriving the form of the theory in Section 4.1 and devote the major part of the section to the derivation and to the study of the insertion in Section 4.2.

\subsection{Constructing the form of the theory}

Let us start by writing the free action for the NS sector. The equation of motion we are after is

$$
\tilde{Q} \Psi=0
$$

and the action should be invariant under the gauge transformation

$$
\delta \Psi=\tilde{Q} \Lambda .
$$

From the discussion of the previous section we infer that $\Psi$ lives in the large Hilbert space, has ghost number one and its picture number is either bounded, or unbounded with some (unknown) restriction on the behaviour of $\Psi$ 's components as a function of the picture number. It is natural to use $\Psi \tilde{Q} \Psi$ for the construction of the free action. Since the string field lives in the large Hilbert space, we have to use the large Hilbert space CFT expectation

\footnotetext{
${ }^{10}$ The idea that in some cases all pictures should contribute is not new [31]. However, we suggest that it "might be a feature, not a bug."
} 
value for the integration over the space of string fields. We can also consider some linear operation to be performed before the integration. Hence, the free action should be of the form

$$
S_{\text {free }}=-\frac{1}{2} \oint \mathcal{O}(\Psi \tilde{Q} \Psi),
$$

where the action has been written with a canonical normalization despite the fact that we have not specified $\mathcal{O}$ yet.

From the experience we have with other theories, we know that the equation of motion and gauge symmetry can be naturally extended to the nonlinear level by writing

$$
S=-\oint \mathcal{O}\left(\frac{1}{2} \Psi \tilde{Q} \Psi+\frac{1}{3} \Psi^{3}\right) .
$$

The infinitesimal gauge symmetry related to this action is

$$
\delta \Psi=\tilde{Q} \Lambda+[\Psi, \Lambda],
$$

and its finite form is

$$
\Psi \rightarrow \mathrm{e}^{-\Lambda}(\tilde{Q}+\Psi) \mathrm{e}^{\Lambda} .
$$

An important consequence of the above is that if the original picture number is allowed to be non-zero, the picture number cannot be bounded and we are led to consider the space of (properly restricted) string fields with arbitrary picture number.

\subsection{Constructing the mid-point insertion}

One might wonder whether a non-trivial $\mathcal{O}$ is really needed. Let us give two arguments in favour of a non-trivial $\mathcal{O}$ :

- The ghost number of $\Psi \tilde{Q} \Psi$ equals three, but the integration we are using is the large Hilbert space integration, which is non-vanishing only for ghost number two string fields. Hence, the action would be identically zero without an $\mathcal{O}$ insertion and it would not imply the desired equation of motion.

- The large Hilbert space integral picks up the copy of the small Hilbert space that is multiplied by $\xi_{0}$. Nevertheless, as we showed in the previous section, it is the small Hilbert space without this insertion that carried the physical information when $\tilde{Q}$ is used. 
The only consistent form for $\mathcal{O}$ is that of a mid-point insertion of a zeroweight conformal primary. ${ }^{11}$ The remarks above suggest that this insertion should contain the $\xi$-field. Another immediate restriction on $\mathcal{O}$ is that it has to commute with the kinetic operator, since otherwise the equations of motion and gauge transformations will not work out correctly,

$$
\tilde{Q} \mathcal{O}=0 .
$$

Decomposing the mid-point insertion with respect to the picture number

$$
\mathcal{O}=\sum_{n \in \mathbb{Z}} \mathcal{O}_{n}
$$

turns the relation (4.7) into a recursion relation for the $\mathcal{O}_{n}$ 's,

$$
Q \mathcal{O}_{n}=\eta_{0} \mathcal{O}_{n+1} .
$$

In order to be able to use the recursion relations, an initial condition is also needed. For finding an appropriate initial condition we invoke the "correspondence principle." Let us fix the picture-related gauge symmetry by restricting the string field to carry zero picture number and to live in the small Hilbert space. This partial gauge fixing reduces the action to that of the modified theory, provided that we choose

$$
\mathcal{O}_{-1}=\xi Y_{-2}=\mathcal{Y},
$$

where $\mathcal{Y}$ is given by (3.16). This choice of $\mathcal{O}_{-1}$ implies that all the classical solutions of the modified theory $[22-24]$ are also solutions, with the same action and cohomology, of the new theory. Moreover, it is also possible to generalize the construction of boundary states [32] to the modified theory [17] and thus, also to our case. All that gives much credibility to the construction.

Substituting $\mathcal{O}_{-1}$ into the recursion relation (4.9) immediately leads to

$$
\begin{aligned}
& \mathcal{O}_{0}=\xi Y=P, \\
& \mathcal{O}_{1}=\xi
\end{aligned}
$$

We see that in the three examples above, the insertion is given by the product of $\xi$ and the picture-changing operators $Y_{-2}, Y, 1$. This is the case since the

\footnotetext{
${ }^{11}$ We refer, again, to section 2 of [15], for discussion on this subject. Also, note that there are two "mid-points," due to the doubling trick. One may hope at this stage that both $z= \pm \mathrm{i}$ will be proven to be equivalent or that some specific superposition of the two will be forced on us by requiring reality of the action, or by some other principle.
} 
picture-changing operators themselves are invariant under $Q$ and $\eta$, while upon acting on $\xi, Q$ produces the picture- changing operator $X$. This state of affairs cannot continue to $|p|>1$ picture numbers without a modification, due to the OPE singularities (3.19). A straightforward resolution is to consider only the non-singular part ${ }^{12}$

$$
\mathcal{O}_{n}= \begin{cases}\oint_{w} \frac{d z}{2 \pi \mathrm{i}} \frac{\xi(z) X_{n-1}(w)}{z-w}, & n>1, \\ \oint_{w} \frac{d z}{2 \pi \mathrm{i}} \frac{P(z) Y_{n}(w)}{z-w}, & n<-1\end{cases}
$$

where $X_{p}$ and $Y_{p}$ are the multi-picture-changing operators.

One might wonder whether this construction is reliable, e.g., whether multi-picture-changing operators exist at all and whether they are unique in some sense. In fact they are. To explain this claim, let us recall some more known facts about the RNS string [33,34]:

- The Becchi-Rouet-Stora-Tyutin (BRST) operator $Q$ commutes with the ghost and picture generators. Hence, the cohomology can be decomposed to definite ghost and picture numbers.

- The cohomologies at the same ghost numbers and different picture numbers are isomorphic.

- For non-zero momentum the cohomology is concentrated in ghost numbers one and two, which are in fact (Poinearè) dual.

- Unique non-trivial elements exist in the cohomologies at zero momentum at ghost numbers zero and three (and all picture numbers).

These facts imply that picture-changing operators exist for all integer picture numbers. We can also deduce that these multi-picture-changing operators are unique, up to the addition of $Q$-exact terms. We denote these operators by $X_{n}$, that is we define

$$
X_{n} \equiv \begin{cases}X_{n}, & n>0 \\ 1, & n=0 \\ Y_{n}, & n<0\end{cases}
$$

The OPE $X_{n} X_{m}$ might generally contain singular terms. These singular terms, however, must be $Q$-exact, as can be seen from plugging the OPE

\footnotetext{
${ }^{12}$ Note, that the fact that our insertion includes all possible pictures is important, since it enables a non-trivial value for the action for string fields of arbitrary picture number.
} 
into a general expectation value and using the observations above. For similar reasons, the regular term must equal $X_{n+m}$,

$$
X_{n} X_{m}=X_{n+m}+Q \text {-exact. }
$$

Even more explicitly we may write

$$
X_{n}(w) \equiv \begin{cases}\oint_{w} \frac{d z}{2 \pi \mathrm{i}} \frac{X_{n-1}(z) X(w)}{z-w}, & n>1, \\ \oint_{w} \frac{d z}{2 \pi \mathrm{i}} \frac{Y_{n+1}(z) Y(w)}{z-w}, & n<-1 .\end{cases}
$$

With the definitions (4.12), (4.13) and (4.15), the insertion we propose can be schematically written as

$$
\mathcal{O} \simeq \xi \sum_{n=-\infty}^{\infty} X_{n}
$$

It is amusing to notice that if we use (4.14) for replacing $X_{n}$ by $X^{n}$, forgetting for the moment about the ( $Q$-exact) OPE divergences and about convergence radius issues, we can write

$$
\mathcal{O} \simeq \xi\left(\frac{1}{1-X}+\frac{1}{1-X^{-1}}-1\right)=0 .
$$

A more relevant and more accurate observation is that, in the language of $[25,35]$, our insertion is the "unique $U(1)$-neutral extension of the vertex operator 1." This is a very natural insertion for a democratic-picture formalism. While we do not think that this is of a particular importance, we note that the proposed mid-point insertion probably does not have a non-trivial kernel. The existence of a regular string field whose OPE with $\mathcal{O}$ is zero at all the picture numbers seems to us very unlikely.

So far we were ignoring the fact that $\mathcal{O}$ has to be a primary field. We would now like to prove that a primary solution to the recursion relation exists. As a bonus, we will also prove that all the picture-changing operators have primary representatives. The proof goes by induction for $p>1$ and for $p<0$. The respective initial conditions are $\mathcal{O}_{1}=\xi$ and $\mathcal{O}_{0}=P$, which are completely fixed by their quantum numbers and are indeed primaries. Acting on a zero-weight primary field with either $Q$ or $\eta_{0}$ gives back a primary. Hence, we can assume that the multi-picture-changing operators are primaries and we only have to prove that the $\mathcal{O}_{p}$ 's are also primaries. 
Given that $\mathcal{O}_{p}$ is primary for some $p>0$, it follows from (4.12) and (4.15) that $X_{p}=Q \mathcal{O}_{p}$ is also primary. The $\mathcal{O}_{p+1}$ defined by (4.12) is not a primary. In fact, for $n \geq 0$ we can write

$$
\begin{aligned}
L_{n} \mathcal{O}_{p+1} & =\oint \frac{d z}{2 \pi \mathrm{i}} z^{n+1} \frac{d w}{2 \pi \mathrm{i}} T(z) \frac{\xi(w)}{w} X_{p}(0) \\
& =\oint \frac{d w}{2 \pi \mathrm{i}} w^{n} \partial \xi(w) X_{p}(0)=-n \xi_{n} X_{p}(0) .
\end{aligned}
$$

Here, the first equality is a mere substitution. In the second equality we used the fact that $X_{p}$ is a primary. Integration by parts leads to the final result. We note that while $\mathcal{O}_{p+1}$ is not a primary, the problematic terms introduced by acting on it with the Virasoro operators all lie in the small Hilbert space. Hence, we could try to cancel them by adding to $\mathcal{O}_{p+1}$ a term, which resides in the small Hilbert space. Such a term would not lead to a change of $X_{p}$, so the recursion relations at lower orders are intact. Let us prove that such a term exists.

Let $\mathcal{S}$ be the set of non-zero elements obtained from repeatedly acting on $\mathcal{O}_{p+1}$ with positive Virasoro operators. The level of this set is bounded by some integer $N$. Define $\mathcal{S}_{N}$ to be the set of all possible level- $N$ combinations of Virasoro operators acting on $\mathcal{O}_{p+1}$,

$$
\mathcal{S}_{N}=\left\{L_{N} \mathcal{O}_{p+1}, L_{N-1} L_{1} \mathcal{O}_{p+1}, \ldots\right\} .
$$

Some of the elements of $\mathcal{S}_{N}$ are actually zero, but let us consider the more general case, in which the $\mathcal{O}_{p+1}$ operator is replaced by an arbitrary (nonprimary) zero-weight operator, whose maximal level under the action of multiple Virasoro operators is $N$. The size of $\mathcal{S}_{N}$ is $P(N)$, the number of partitions of $N$ into positive integers. Let $\mathcal{C}_{N}$ be the set of all elements that can be formed by acting on $\mathcal{S}_{N}$ with an arbitrary number of negative Virasoro operators of total level $-N$. The set $\mathcal{C}_{N}$ contains $P(N)^{2}$, a priori independent, zero-weight, small Hilbert space elements. These elements are annihilated by the action of Virasoro operators of total level greater than $N$, but not by operators of level $N$ or lower. Consider now

$$
\mathcal{O}_{p+1}^{(N)}=\mathcal{O}_{p+1}+\sum_{k=1}^{P(N)^{2}} \alpha_{k} C_{k},
$$

where $C_{k} \in \mathcal{C}_{N}$ and the $\alpha_{k}$ 's are coefficients. Act on $\hat{\mathcal{O}}_{p+1}$ by all $(P(N))$ possible level $N$ Virasoro operators and require that the result vanishes. This gives $P(N)$ equations, where each equation contains the $P(N)$ elements of $\mathcal{S}_{N}$, which should vanish separately. All in all, we obtain $P(N)^{2}$ linear 
non-homogeneous equations in $P(N)^{2}$ variables. In fact, it is easy to see that the matrix describing these $P(N)^{2}$ equations factorizes into $P(N)$ identical blocks of size $P(N) \times P(N)$. Each block is nothing but the level- $N$ Kacmatrix with $c=0$ and $h=-N$. The Kac determinant at level $N$ equals up to a constant to the product of terms of the form

$$
P_{r, s}=h-h_{r, s}, \quad h_{r, s}=\frac{c-1}{24}+F_{r, s}^{2},
$$

with $F_{r, s}^{2}$ known, $c$-dependent positive constants. In our case $h$ is a negative integer, while

$$
h_{r, s} \geq-\frac{1}{24}
$$

Hence, the Kac-determinant is non-zero and a solution exists and is unique. The solution defines $\mathcal{O}_{p+1}^{(N)}$ as a zero-weight operator that differs from our original $\mathcal{O}_{p+1}$ only in the small Hilbert space. Acting on $\mathcal{O}_{p+1}^{(N)}$ with Virasoro operators of total level greater than $N-1$ gives zero by construction. We can now repeat the procedure, defining $\mathcal{O}_{p+1}^{(N-1)}$ that removes the $N-1$ terms and so on. The operator $\mathcal{O}_{p+1}^{(1)}$ is then primary by construction. The fact that many of the elements of $\mathcal{C}_{N}$ are zero does not modify any of the arguments we made and for $p>1$ the proof is completed. The proof for $p<0$ is almost identical. All that is needed is to replace $Q$ by $\eta_{0}, \xi(z)$ by $P(z)$ and the small Hilbert space by the dual small Hilbert space.

While our proof implies using $P(N)^{2}$ elements for eliminating $\mathcal{S}_{N}$, we can use the explicit form (4.18) in order to work in practice with smaller sets. In fact, generalizing (4.18), we see that all the elements of $\mathcal{S}_{N}$ are equal up to a constant. Hence, we can replace the $P(N)^{2}$ elements of $\mathcal{C}_{N}$ by the $P(N)$ elements obtained by acting on $\xi_{N} X_{p}(0)$ with total level $-N$ Virasoro operators. We now obtain a set of at most $P(N)$ equations with at most $P(N)$ variables. The matrix of coefficient is again the Kac matrix, which leads to a unique solution. This is a simplified (but less general) version of the theorem above.

Let us now consider $\mathcal{O}_{2}$ as an example. The theorem implies that it is given by

$$
\mathcal{O}_{2}=-c \xi \xi^{\prime}+\xi \mathrm{e}^{\phi} G_{m}+\left(2 b \eta \xi \phi^{\prime}+\eta \xi b^{\prime}-2 b \xi \eta^{\prime}\right) \mathrm{e}^{2 \phi}+\tilde{\mathcal{O}}_{2},
$$

where $\tilde{\mathcal{O}}_{2}$ resides in the small Hilbert space. Here, we truncated the expression one obtains using (4.12) to its $\xi_{0}$ component, as the rest of it lies in 
the small Hilbert space, which we can freely change. Applying the Virasoro operators, we see that $N=2$ in this case and that

$$
-L_{1}^{2} \mathcal{O}_{2}=L_{2} \mathcal{O}_{2}=4 b \mathrm{e}^{2 \phi} .
$$

Considering the two terms $L_{-1}^{2} b \mathrm{e}^{2 \phi}$ and $L_{-2} b \mathrm{e}^{2 \phi}$ gives two equations, which we can solve. The solution, however, is quite cumbersome when written explicitly. Moreover, while the solution is universal by construction, it is not manifestly universal, e.g., for the standard matter fields we obtain different coefficients of $T_{X}$ and $T_{\psi}$ in various terms of the solution. A way around it is to note that in order to prove the theorem, we do not really have to use the full energy momentum tensor. All that is needed is to have $T_{\xi \eta}$, as well as the part of the energy momentum tensor associated with the fields that appear in (4.24), i.e., we can work with $T_{g}$ instead of with the total energy momentum tensor in the case at hand. Working with $T_{g}$ gives again two equations. Solving the equations leads to a $\tilde{\mathcal{O}}_{2}$, which contains six, manifestly universal terms. Actually, playing a bit with coefficients reveals an even simpler solution

$$
\tilde{\mathcal{O}}_{2}=-\left(29 b^{\prime \prime}+51 b^{\prime} \phi^{\prime}+2 b \phi^{\prime 2}\right) \frac{\mathrm{e}^{2 \phi}}{86} .
$$

This illustrates the fact that while a solution of the form used in the proof above is unique, there are generally many other (universal) solutions. It is interesting to note that although we explicitly removed only the second-order poles, we got a primary field, without actually going to the next stage.

It is now straightforward to calculate $X_{2}$, which we did in a particular background (one-dimensional linear dilaton) for simplicity. The resulting expression is a sum of 150 terms (in this background) and is not particularly illuminating. This $X_{2}$ is primary by construction and can be used to define $\mathcal{O}_{3}$. Now, $N=6$ and terms with various $\phi$-momenta appear. The $\mathrm{e}^{4 \phi_{\text {-terms }}}$ are found all the way to $N=6$, while the $\mathrm{e}^{3 \phi_{\text {-terms }}}$ and $\mathrm{e}^{2 \phi_{-}}$ terms have $N=4$ and 3 , respectively. It is clear that all these terms would not be removed in a single step. We examined a 371-parameter universal ansatz, containing the terms that are dictated by the theorem, and found a 94-parameter family of solutions. In a relatively simple case a primary $\mathcal{O}_{3}$ can be written as a sum of 336 terms.

Having settled the issue of being primary, we now want to discuss the amount of freedom in solving the recursion relation (4.9), without the restriction to primary operators. Too much freedom in choosing $\mathcal{O}$ would lead to an embarrassment of riches, i.e., having several, presumably inequivalent, theories. This state of affairs was exactly one of the grounds for criticizing 
the modified theory. Then, in [17], we showed that, at least classically, all these theories are equivalent, regardless of the exact form of the insertion and regardless of the distribution of the insertion among the two mid-points $( \pm \mathrm{i})$. The only thing we need in order to make the same assertion here is to show that the difference $\delta \mathcal{O}$ of two candidate $\mathcal{O}$ insertions is given by a $\tilde{Q}$-exact term. It is clear from (4.7) that

$$
\tilde{Q} \delta \mathcal{O}=0
$$

However, while $Q$ and $\eta_{0}$ are separately trivial in the large Hilbert space, their difference, $\tilde{Q}$, is not. Hence, this equation is not enough for our proof.

Nonetheless, the initial condition for the non-unique $\mathcal{O}_{-1}$ fixes the insertions $\mathcal{O}_{0}$ and $\mathcal{O}_{1}$ uniquely (4.11), at least as long as we are considering only chiral insertions. This can be seen simply by examining all possible insertions with those quantum numbers. Consider now $\mathcal{O}_{2}$. The recursion relation (4.9) implies that it is defined up to an $\eta_{0}$-closed term. Since $\eta_{0}$ is exact in the large Hilbert space, we can write

$$
\delta \mathcal{O}_{2}=-\eta_{0} \Upsilon_{3}
$$

This implies at the next picture number the identity

$$
\eta_{0} \delta \mathcal{O}_{3}=Q \mathcal{O}_{2}=\eta_{0} Q \Upsilon_{3}
$$

whose general solution is

$$
\delta \mathcal{O}_{3}=Q \Upsilon_{3}+\eta_{0} \Upsilon_{4}
$$

We see that the total effect of $\Upsilon_{3}$ is to induce a change in $\mathcal{O}$,

$$
\delta \mathcal{O}=\tilde{Q} \Upsilon_{3}
$$

and the analysis can then be repeated for $\Upsilon_{4}$ and so on. A similar analysis can be applied for negative picture numbers. We conclude that, in general,

$$
\delta \mathcal{O}=\tilde{Q} \Upsilon
$$

Hence, all the theories that are defined by solutions of the recursion relation (4.9) with the initial conditions (4.11) are classically equivalent. This also implies that at least for the purpose of evaluating the action of a classical solution, we do not have to find an explicit primary conformal representative of $\mathcal{O}$. The regular part of (4.16) will do. Note, however, that the two parts 
of the action would have to be evaluated in the same coordinate system. This is not really a restriction, since for solutions one simply evaluates

$$
S=-\oint \mathcal{O}\left(\frac{1}{2} \Psi \tilde{Q} \Psi+\frac{1}{3} \Psi(-\tilde{Q} \Psi)\right)=-\frac{1}{6} \oint \mathcal{O} \Psi \tilde{Q} \Psi .
$$

Then, it is possible to perform the calculations using (4.15) rather than with the cumbersome explicit expressions of the conformal primaries.

\section{The Ramond sector and general D-brane systems}

In order to complete this work, we have to incorporate the Ramond sector into the formalism. This sector leads to various singularities within the modified theory. Nonetheless, the construction of the action of the modified theory, as well as of Witten's theory, relied on correct principles. Hence, if we could manage to construct an action that generalizes these constructions, without suffering from their problems, we would know that we are on the right track. Thus, we search for an action that reduces to

$$
S=-\int Y_{-2}\left(\frac{1}{2} \Psi Q \Psi+\frac{1}{3} \Psi^{3}\right)-\int Y\left(\frac{1}{2} \alpha Q \alpha+\Psi \alpha^{2}\right),
$$

when the NS and Ramond string fields live in the small Hilbert space and carry picture numbers 0 and $-\frac{1}{2}$, respectively. From the inconsistency of the modified theory we can infer that the above set of restrictions does not form a consistent set of gauge conditions. We return to the issue of gauge fixing after the theory is constructed.

We know already that the first part of this action generalizes to (4.4). Then, applying the same philosophy as before, we replace $Q$ with $\tilde{Q}$, the $Y$ insertion with $\mathcal{O}^{R}$ and the integration with integration in the large Hilbert space. The correspondence principle implies that

$$
\mathcal{O}_{0}^{R}=P .
$$

Having $\tilde{Q}$ as the kinetic operator implies again the recursion relations (4.9) and hence

$$
\mathcal{O}^{R}=\mathcal{O}^{\mathrm{NS}} .
$$

It is straightforward to see that the resulting action can be simply written in terms of a single string field and that it takes exactly the simple form (4.4), 
provided we redefine

$$
\Psi \rightarrow \Psi+\alpha
$$

Thus, we unified the NS and Ramond string fields. The resulting string field $\Psi$ includes all possible integer (NS) and half-integer $(R)$ picture numbers at ghost number one. The full gauge symmetry of the action is (4.5), where the even gauge string field $\Lambda$ carries ghost number zero and an arbitrary integer (NS) or half-integer $(R)$ picture number. There are no picture-changing operators or other mid-point insertions in the definition of the gauge symmetry. Hence, no singularities can emerge and the gauge symmetry is well defined.

The inclusion of the various sectors ( $\mathrm{NS} \pm, R \pm$ ) of a general D-brane configuration, described by some Chan-Paton factors, is straightforward and should be done in the same way as in [11] (see also [9]), i.e., the space of string fields should be tensored with a matrix space representing the Chan-Paton factors, as well as with the "internal Chan-Paton space" of two by two matrices. The NS+ string field is tensored with an internal Chan-Paton factor of $\sigma_{3}$ (granted also to $Q$ ), while the NS- string field is tensored with $\mathrm{i} \sigma_{2}$. Since the Ramond string fields are added to the NS+ string field in our formulation, they should also be tensored with $\sigma_{3}$. A single Chan-Paton entry cannot contain both $R$ sectors. Hence, there is no problem in assigning the same factor to both $R \pm$. This assignment is also consistent with our discussion on this subject in [16]. The fact that $Q$ gets a $\sigma_{3}$ factor implies that $\eta_{0}$ gets the same factor (again, in accordance with the discussion in $[16,24])$. This implies that $\xi$ and $P$ insertions should also be tensored with $\sigma_{3}$, which suggests that the whole $\mathcal{O}$ insertion is to be tensored with this factor. This, in a sense, clarifies the origin of the $\sigma_{3}$ insertion on $Y_{2}$ in the modified theory: It is a remnant from integrating the $\xi$ insertion that has to carry this factor, when going from the large Hilbert space to the small one. The gauge string fields should all carry a unity factor, except the NS- gauge string field that carries a $\sigma_{1}$ factor. With these assignments all the axioms needed continue to hold and all sectors of an arbitrary (not necessarily BPS) D-brane system can be uniformly and covariantly described.

\section{Field-antifield formulation and gauge fixing}

The gauge symmetry of our theory is infinitely reducible and closes only "using the equations of motion," i.e., only up to trivial gauge transformations. This state of affairs calls for the use of the (covariant) field-antifield 
(BV) formalism [36-40] (see [41-43] for reviews). This formalism replaces the gauge symmetry by a BRST symmetry, which can be fixed at a later stage using a "gauge-fixing fermion." Generally speaking, the BV construction is nothing but trivial. Luckily, the algebraic structure at hand is identical to that of the bosonic theory. The BV formulation of this theory was studied by Thorn $[44,47]$ and by Bochicchio $[45,46]$. All what we have to do then is to use the following substitutions:

$$
Q_{\mathrm{bos}} \rightarrow \tilde{Q}=Q_{\mathrm{RNS}}-\eta_{0}, \quad \int_{\mathrm{bos}} \rightarrow \oint \mathcal{O}, \quad \Psi_{\mathrm{cl}, \mathrm{bos}} \rightarrow \Psi_{\mathrm{cl}, \mathrm{RNS}}
$$

where as already explained, the classical RNS field $\Psi_{\text {cl,RNS }}$ carries ghost number one and all (integer and half-integer) picture numbers. Mimicking the construction of [44-47] for the case at hand is straightforward, due to the identical algebraic structure (the properties of $\tilde{Q}$ and $\oint \mathcal{O}$ as well as the form of the redundant gauge symmetry). The construction leads (before gauge fixing) to an action identical in form to (4.4), only with the string field $\Psi_{\mathrm{cl}, \mathrm{RNS}}$ replaced by the string field $\Psi_{\mathrm{BV}, \mathrm{RNS}}$, which contains all possible picture and ghost numbers, as well as both (NS and $R$ ) sectors of the theory. This is very satisfactory from an aesthetic point of view, since now the theory is defined by a string field that uses the whole "Hilbert space." Moreover, for the case of a general D-brane system, the string field lives in the maximal space (in terms of sectors and ghost and picture numbers) consistent with the system.

One possible subtlety with this construction is the implicit assumption that the integration measure that we use induces a non-degenerate inner product in the space of string fields. While the analogous assertions in some other cases are pretty much CFT axioms, our case might depend on the definition of the space of string fields. The reason for the difference is that, in theories with no explicit insertions, the space is naturally decomposed into subspaces with fixed ghost and picture numbers and with fixed conformal weights. Each such space is dual to another such space with respect to the inner product. Hence, the string field can be decomposed into a direct sum of spaces and within each of these spaces it can be further decomposed into a finite sum of component fields. Each component field has as its anti-field another component field that lives in its dual space. All that implies that the BV theory can be equally well formulated in terms of the component fields and in terms of string fields.

When mid-point insertions are included, they tend to couple fields with various conformal weights. Moreover, in our case, there is a decomposition only with respect to the ghost number, while all picture numbers are coupled, due to the presence of the $\mathcal{O}$ insertion, which includes all integer picture 
numbers. The inner products between the elements of two dual spaces at ghost numbers $g$ and $3-g$ can be collected into an infinite matrix. The question of degeneracy then depends on the space of allowed vectors and dual vectors (ghost number $g$ and $3-g$ string fields, respectively). One observation is that this matrix contains infinitely many infinite size blocks, of constant entries. One could fear that these blocks, originating from sets of physically equivalent vertex operators, imply that the measure is degenerate. This is not necessarily the case, since the elements generating such a block couple also to other, in particularly off-shell, states.

We would like to stress that it would be wrong to try and "invert" $\mathcal{O}$ treating it separately from the measure, as it is often done with the $Y_{-2}$ of the modified theory. Particularly, the inverse might not be defined over the correct space of string fields, i.e., the formal object " $\mathcal{O}^{-1} \Psi$ " would probably not be part of the space of string fields for any non-zero $\Psi$.

We believe that the subtleties related to the definition of the space of string fields have a resolution, the BV construction is reliable and can be used for gauge fixing. To that end, a gauge-fixing fermion should be introduced. The gauge-fixing fermion is an odd functional of the string fields, with (second quantized) ghost number -1 . It depends on some auxiliary fields, e.g., non-minimal sets of variables that serve as Lagrangian multipliers. The set of non-minimal fields needed for our case is known [43]. However, there are many ways for constructing gauge-fixing fermions, not all of which are admissible. Moreover, it is not clear whether the subtleties with the quantum master equation of the bosonic theory persist in our theory. Even if it would be possible to show that the quantum master equation holds in our case, the construction of an admissible gauge-fixing fermion would probably still be non-trivial. In calculating RNS loop scattering amplitudes subtleties appear related to the measure on supermoduli spaces [48-50]. It might be too optimistic to expect that these issues can be avoided by using string field theory for the evaluation of amplitudes. On the other hand, if one could show that superstring field theory is consistent at the quantum level, this could be an alternative definition for the superstring, not relying on the subtleties of supermoduli spaces! We leave the resolution of these important questions to future work.

A somewhat naive alternative to the discussions above would be to simply enforce some auxiliary conditions that suppose to remove the gauge-related redundancy. For example, when the theory is restricted to the NS sector, it is possible to constrain the string field to carry zero picture number and to live in the small Hilbert space. The action then reduces to that of $[5,7]$, where a further gauge fixing is needed, e.g., Siegel gauge, Schnabl gauge, a linear $b$-gauge [51], or an $a$-gauge $[52,53]$. Trying to restrict the NS sector 
to other picture numbers seem to lead to inconsistent results. At picture number -1 , one might argue that Witten's theory is obtained, while at other picture numbers other inconsistent theories seem to emerge. ${ }^{13}$ It would be very interesting to consider universal gauge fixings that do not concentrate at fixed picture numbers. We leave this interesting project to future work.

\section{Supersymmetry}

Up to this point, our discussion was universal, i.e., it did not depend in any way on the BCFT used in the definition of the theory. Now, however, we would like to study supersymmetry within our theory, which is background dependent. Hence, we specify that we work in the standard ten-dimensional RNS flat space, i.e., the matter system is composed of ten world-sheet scalars $X^{\mu}$ and ten world-sheet fermions $\psi^{\mu}$.

The space-time supersymmetry generators of the RNS formalism carry half-integer picture numbers. In a fixed picture number theory this implies that picture-changing operators should be appended to the definition of the supersymmetry transformation. For consistency, these operators have to be inserted at the string mid-point, which leads to singularities and probably takes the string field outside its domain of definition. ${ }^{14}$ Working at an unrestricted picture-number space, as we do here, potentially avoids this problem.

In previous cubic formulations [3], supersymmetry was generated by the zero momentum, integrated, $-\frac{1}{2}$-picture, fermion vertex. We may consider the same generator in our theory,

$$
\delta_{\mathrm{SUSY}} \Psi=\epsilon^{\alpha} Q_{\alpha} \Psi \equiv Q^{\epsilon} \Psi, \quad Q_{\alpha}=\oint \frac{d z}{2 \pi \mathrm{i}} \mathrm{e}^{-\frac{\phi}{2}} S_{\alpha}(z)
$$

\footnotetext{
${ }^{13}$ After the first version of this paper appeared, it was discovered in [54] that gauge fixing the theory to picture number -1 does not lead to Witten's theory, but to another, classically consistent, theory, which is the $\mathbb{Z}_{2}$ dual of the modified theory in the sense of $[25,55]$. Moreover, it was found there that the democratic theory can be reduced using another gauge fixing to the non-polynomial theory and that this partial gauge fixing can also be extended to the Ramond sector. Finally, it is also argued there that gauge fixing at other fixed picture numbers is probably inconsistent. These new results give strong evidence in favour of the democratic theory.

${ }^{14}$ We stress again that general mid-point operator insertion on the string field might lead to singularities. In order to avoid these potential problems one has to restrict somehow the space of string fields, such that potentially harmful mid-point insertions would not be allowed. This in turn implies that mid-point operator insertions in the action, as we consider here, are harmless.
} 
Here, $S_{\alpha}$ is the spin field, which is responsible for exchanging the NS and Ramond sectors while $\epsilon^{\alpha}$ are odd parameters. Using the integrated vertex seems to be the better option, since the unintegrated vertex should be inserted at a given point. This point cannot lie inside the local coordinate patch, since that might lead to singularities from collisions with the state itself. It also cannot be inserted outside the local coordinate patch, as that might take the string field outside of its domain of definition, as well as to introduce singularities from multiplication by other string fields.

The generator (7.1) would be a symmetry, provided that the following three conditions hold $[2,3]$ :

(1) $Q^{\epsilon}$ should be a derivation of the star product,

$$
Q^{\epsilon}(A B)=Q^{\epsilon} A B+A Q^{\epsilon} B .
$$

(2) $Q^{\epsilon}$ should be invariant under the kinetic operator,

$$
\left[\tilde{Q}, Q^{\epsilon}\right]=0
$$

(3) $Q^{\epsilon}$ should leave the integration measure invariant,

$$
\oint \mathcal{O} Q^{\epsilon} A=0 \quad \forall A
$$

The first condition holds, since $Q^{\epsilon}$ is an integral of a current. The second condition holds, since the vertex defining $Q^{\epsilon}$ lives in the small Hilbert space and is on-shell. The third condition, however, does not hold, since the vertex has singular OPEs with many of the $\mathcal{O}_{n}$ 's. This is hardly surprising, since these operators are essentially picture-changing operators, which should act non-trivially on an on-shell vertex at any given picture.

While our supersymmetry generators fail to be symmetries off-shell, they are symmetries on-shell. By that we do not mean that the action around solutions is invariant under the linearized supersymmetry transformation: the action is linearly invariant under any change of a solution by definition. We mean that this linearized transformation naturally acts on the space of solutions, i.e., it sends solutions to solutions,

$$
\delta_{\mathrm{SUSY}}\left(\tilde{Q} \Psi+\Psi^{2}\right)=\tilde{Q} Q^{\epsilon} \Psi+Q^{\epsilon} \Psi \Psi+\Psi Q^{\epsilon} \Psi=Q^{\epsilon}\left(\tilde{Q} \Psi+\Psi^{2}\right)=0
$$

Here, use was made of the properties (7.2a) and (7.2b). 
Nonetheless, a genuine symmetry must be defined also off-shell. ${ }^{15}$ One possible direction towards defining supersymmetry off-shell is to consider a superposition of supersymmetry generators at various picture numbers

$$
Q_{\alpha} \stackrel{?}{=} \oint \frac{d z}{2 \pi \mathrm{i}} \sum_{p \in\left(\mathbb{Z}+\frac{1}{2}\right)} k_{p} V_{\alpha}^{p}(z)
$$

where $k_{p}$ are unknown coefficients. These coefficients are restricted by the requirement that $(7.2 \mathrm{c})$ holds. One might think that this restriction gives a set of recursion relations, similar to the ones that we got for the $\mathcal{O}_{n}$ insertions. Instead, one gets an infinite set of equations, each one including infinitely many summands. Each summand can include many different operators that should all independently vanish by the choice of coefficients. It is neither clear to us whether this system of equations has a solution, nor how to construct this solution perturbatively, or otherwise.

A more promising approach can be based on the fact that (when integrated) the fermion vertex is $\tilde{Q}$-closed. Our experience with string field theory from the last few years suggests that it might be useful to write it formally as if it were exact [56-58]. We propose to write the integrated fermion vertex as

$$
V_{\alpha}=-\tilde{Q} W_{\alpha} .
$$

Being loyal to the democratic paradigm, the above vertex should be allowed to have an arbitrary picture and choosing different pictures should result in gauge equivalent configurations. We would then write

$$
Q^{\epsilon} \Psi=\tilde{Q}\left(W^{\epsilon}\right) \Psi .
$$

The problem is that the string field is not necessarily closed, so (7.2c) is still not obeyed, which is not surprising since all we did was to rewrite the form of the vertex. The following modification can be proposed in order to resolve this problem:

$$
\delta_{\mathrm{SUSY}} \Psi \stackrel{?}{=} \tilde{Q}\left(W^{\epsilon} \Psi\right)
$$

\footnotetext{
${ }^{15}$ The situation we have should not be confused with the common one of having a symmetry algebra that "closes only up to the use of the equations of motion." In the case at hand, we cannot even claim that we have a symmetry.
} 
Both (7.6) and (7.7) agree when restricted to vertex operators and reduce in this case to the standard expression. However, while (7.2c) holds for (7.7), (7.2a) no longer holds. The natural way to resolve this problem is to notice that (7.7) is in fact the linearized form of an infinitesimal fermionic gauge transformation. ${ }^{16}$ Hence, it is natural to add to the above a non-linear term and define

$$
\delta_{\mathrm{SUSY}} \Psi=\tilde{Q}\left(W^{\epsilon} \Psi\right)+\left[\Psi, W^{\epsilon} \Psi\right]
$$

Relations (7.2) are not obeyed now, but there is no reason for them to be obeyed, since (7.8) is not a linear transformation of the string field. It is nevertheless a symmetry, as it is a formal gauge symmetry (recall that we use a formal gauge string field), ${ }^{17}$ with the (formal) gauge string field

$$
\Lambda_{\mathrm{SUSY}}=W^{\epsilon} \Psi
$$

The main potential problem with this proposal is to find an adequate $W^{\epsilon}$, that is, to define it in such a way that while it is a formal string field, the resulting transformation (7.8) defines a genuine string field. Since $V_{\alpha}$ lives in the small Hilbert space, it is possible to write

$$
W_{\alpha} \stackrel{?}{=} \xi V_{\alpha}
$$

This, however, results in an addition to the vertex

$$
\delta V_{\alpha}=-Q W_{\alpha}
$$

i.e., we add to the vertex an expression, which is roughly minus the same vertex in a different picture. This is obviously wrong. Indeed, (7.10) is a genuine string field. Hence, the resulting transformation is a genuine gauge transformation rather than a supersymmetry one. A way out might be to add to $W_{\alpha} \eta$-primitives of all higher pictures. Recall that up to $Q$-exact

\footnotetext{
${ }^{16}$ The idea of formally representing supersymmetry as a gauge symmetry, albeit in a different way, appeared already in [59].

${ }^{17}$ One might have considered $W_{\alpha}=-\int_{-\infty}^{\infty} d z V_{\alpha}(z)|1\rangle$ as the formal gauge string field, with $|1\rangle$ being the identity string field and the integration is in the cylinder coordinates. The gauge string field is manifestly formal due to the presence of the identity string field in its definition and the resulting transformation is exactly (7.1). Nonetheless, this choice is wrong, since the integration limits approach the mid-point insertion, invalidating the arguments that show that a gauge symmetry is a symmetry.
} 
(singular) terms, the recursion relation satisfied by the integrated vertex operators is

$$
V^{p+1}=Q\left(\xi V^{p}\right)+\partial\left(\xi \hat{V}^{p}\right),
$$

where $V^{p}$ is the unintegrated vertex operator, defined by the relation

$$
Q V^{p}=\partial \hat{V}^{p} .
$$

The second term in (7.12) is needed for assuring that the resulting vertex lives in the small Hilbert space and continues to respect (7.13). The recursion relation (7.12) might suffer from OPE singularities. However, as these singularities are exact, they can be safely removed from the definition. Total derivative and $Q$-exact terms could then be added to assure that the operator is primary.

Now, let us define

$$
W_{\alpha}^{p+1}=(-1)^{p-p_{0}} \xi V_{\alpha}^{p}, \quad p+1 \geq p_{0},
$$

and

$$
W_{\alpha}=\sum_{p=p_{0}+1}^{\infty} W_{\alpha}^{p} .
$$

Here $p_{0}$ is an arbitrary starting point. With this definition we obtain

$$
\tilde{Q} W_{\alpha}=-V_{\alpha}^{p_{0}} .
$$

Hence, at the linearized level, the transformation reduces to a supersymmetry transformation. Moreover, the components of $W_{\alpha}$ do not decrease in any way as a function of picture number. Thus, it is indeed a formal gauge string field, as we wanted to have. The difference between starting with two different pictures, on the other hand, is given by a genuine gauge transformation.

The resulting supersymmetry transformation takes the form

$$
\delta_{\mathrm{SUSY}} \Psi=Q^{\epsilon} \Psi-W^{\epsilon}\left(\tilde{Q} \Psi+\Psi^{2}\right) .
$$

The second term vanishes on-shell and the transformation reduces to the standard linear supersymmetry transformation. Off-shell the transformation becomes non-linear with respect to the string field. This might have been expected, since on the one hand string field theory is a non-linear extension 
of the world-sheet formalism, while on the other hand supersymmetry is non-linearly realized in many circumstances.

There is one potential obstacle for our interpretation: It seems that the string field we obtain off-shell is not more genuine than the formal gauge string field. Indeed, our experience with formal gauge string fields suggests that "higher order counter-terms" should be added to the gauge string field in order to obtain a legitimate physical string field. We leave the problem of finding these terms and the related problem of understanding the non-linear terms induced by the supersymmetry algebra in the momentum transformation of off-shell string fields to future study.

\section{Conclusions}

In this work a new universal open RNS string field theory was presented. The theory is cubic and includes a mid-point insertion in the action. This midpoint insertion most probably carries only a trivial kernel. More importantly, while we have an insertion in the action, we allow for mid-point insertions neither in the gauge transformations nor in the equations of motion. Thus, the theory does not suffer from singularities due to collisions of mid-point insertions as was the case with the previous formulations.

The new theory naturally and covariantly unifies the NS and Ramond string fields and can be used to describe open strings on arbitrary D-brane systems. Since it can be reduced to the modified theory, it supports its solutions. These observations give much credibility to the theory.

Nonetheless, there is more to be done. Specifically, one should devise a gauge fixing of the theory, since it is imperative for the construction of perturbation theory, e.g., for defining propagators. While the first step towards that end, i.e., the BV construction was completed and led to an elegant result, it is important to further study this highly non-trivial issue. The understanding of the gauge fixing of our theory might lead not only to further credibility to the theory, but also to new ways of evaluating RNS scattering amplitudes, which avoid the problems with the supermoduli spaces, at least for the scattering of open strings. It is also important to complete the formulation of the off-shell supersymmetry transformations of the theory.

Our construction uses primary multi-picture-changing operators. The existence of these operators was not known and was proven here. Moreover, we showed that within a specific universal space spanned by total Virasoro operators plus a non-trivial piece, these operators are unique. However, when one considers more general, but still universal, spaces, these operators 
are no longer unique. The understanding of multi-picture-changing operators achieved in this work is an important achievement by itself, since it might be of use also for other approaches towards the RNS string.

We used the multi-picture-changing operators in order to construct the (primary) $\mathcal{O}_{n}$ operators, which generalize $\xi$ and $P$ to other picture numbers. The generalization is, however, incomplete, in the sense that while $\xi$ and $P$ serve as contracting homotopy operators for the commuting cohomology operators $Q$ and $\eta_{0}$, we do not know of currents that we can similarly associate with the other $\mathcal{O}_{n}$ operators. It would be interesting if we could define currents $J_{p}(z)$, carrying ghost number one and picture number $p$, such that the $\mathcal{O}_{-p}$ would serve as the contracting homotopy operators for their mutually commuting charges, i.e.,

$$
\begin{aligned}
J_{p}(z) J_{p^{\prime}}(w) & \sim \cdots+\frac{\partial(. .)}{z-w}, \\
T(z) J_{p}(w) & \sim \frac{J_{p}(w)}{(z-w)^{2}}+\frac{\partial J_{p}(w)}{z-w}, \\
J_{p}(z) \mathcal{O}_{-p}(w) & \sim \frac{1}{z-w} .
\end{aligned}
$$

The familiar cases are

$$
J_{0}(z)=J_{B}(z), \quad J_{-1}(z)=\eta(z) .
$$

We managed to find a candidate $J_{-2}$,

$$
J_{-2}=\frac{1}{4}\left(3 c^{\prime \prime}-13 c \phi^{2}+2\left(b c c^{\prime}+c \xi^{\prime} \eta+c T_{m}\right)\right) \mathrm{e}^{-2 \phi} .
$$

In fact, we even found a multi-parameter family of such candidates. There might, however, be further restrictions on $J_{-2}$ coming from consistency with the other $J_{p}$ 's, or otherwise. We believe that a better understanding of these currents (if they exist) might shed light on the nature of the RNS string, as well as help in future string field theoretical research.

Our construction is based on the use of $\tilde{Q}$, which was introduced by Berkovits in [20], for the purpose of relating the RNS formalism and the pure-spinor one $[60,61]$. Here, we used this operator for constructing an RNS string field theory. One might ask whether it is possible to rewrite our theory using pure-spinor variables. This is not straightforward, since the mapping between the two formalisms is for on-shell states only, while we should be looking for an off-shell mapping. On the other hand, a pure-spinor string field theory already exists [13]. This theory is also cubic and it also uses a 
mid-point insertion whose kernel is trivial for the saturation of bosonic zero modes (this is also the origin of the notion of "picture"). However, it uses the non-minimal formulation of pure-spinor string theory [13]. Presumably, it might be related to the present formalism if that would be extended along the lines of $[14,15]$. It would be very interesting to pursue this direction of research, as it might lead to a unified picture of superstring field theory, while clarifying some fundamental issues along the way. A potential obstacle is the fact that the pure-spinor string field should be allowed to be singular with respect to the pure-spinor $\lambda^{\alpha}$, but not too singular. This state of affairs seems to lead to a contradiction that could only be resolved by modifying the pure-spinor formalism itself $[62,63]$. A modification of the pure-spinor formalism should also allow the introduction of a $\mathrm{GSO}(-)$ sector. One approach towards the inclusion of this sector was presented in [64], where some non-minimal sectors were added to the theory. However, it is not clear how to unify these non-minimal sectors with the non-minimal sector of [13]. Presumably, the comparison with the democratic theory might give us some clues regarding the resolution of these difficulties.

We hope that the democratic theory would be found useful for the construction of closed RNS string field theories. Complications in generalizing this work are bound to arise, among other reasons, since in closed string theory the relative cohomologies at different picture numbers are not all isomorphic [65]. Presumably, one could devise a way around this problem by replacing the $b_{0}^{-} \Psi=0$ condition by some sort of a gauge symmetry, in a way analogous to the treatment of $b_{0}$ in the open string case and the treatment of picture number in this work. Another potential difficulty comes from the fact that in the closed string case the mid-point, on which we inserted the operator $\mathcal{O}$, is absent. This difficulty could possibly be resolved along the lines of [66]. This construction could potentially be generalized, using some ideas of the democratic theory and of the NS heterotic string field theory [67], in order to construct the desired closed RNS string field theories as well as a complete RNS heterotic string field theory. This important issue remains for future work.

Identifying correctly the space of string fields is still an open problem even within the context of bosonic string field theory. In fact, some of the most important recent achievements of the field, such as proving Sen's conjectures $[68,69]$ for Schnabl's solution $[26]$ depend crucially on properties of this unknown space $[27,56,70]$. Thus, our proposal that some properties hold for this new theory, provided the space of string fields has some given properties, are along the line of what is currently the common practice. Finding sound definitions for the spaces of string fields of the various string field theories would be highly important, both for understanding these theories, as well as for their proper definition. 


\section{Acknowledgments}

I would like to thank Ido Adam, Ofer Aharony, Nathan Berkovits, Stefan Fredenhagen, Udi Fuchs, Michael Kiermaier, Carlo Maccaferri, Yaron Oz, Stefan Theisen, Scott Yost and Barton Zwiebach for discussions.

This work is supported by the US Department of Energy (D.O.E.) under cooperative research agreement DE-FG0205ER41360. My research is supported by an Outgoing International Marie Curie Fellowship of the European Community. The views presented in this work are those of the author and do not necessarily reflect those of the European Community.

\section{References}

[1] D. Friedan, E.J. Martinec and S.H. Shenker, Conformal invariance, supersymmetry and string theory, Nucl. Phys. B271 (1986), 93.

[2] E. Witten, Noncommutative geometry and string field theory, Nucl. Phys. B268 (1986), 253.

[3] E. Witten, Interacting field theory of open superstrings, Nucl. Phys. B276 (1986), 291.

[4] C. Wendt, Scattering amplitudes and contact interactions in Witten's superstring field theory, Nucl. Phys. B314 (1989), 209.

[5] C.R. Preitschopf, C.B. Thorn and S.A. Yost, Superstring field theory, Nucl. Phys. B337 (1990), 363-433.

[6] I.Y. Arefeva, P.B. Medvedev and A.P. Zubarev, Background formalism for superstring field theory, Phys. Lett. B240 (1990), 356-362.

[7] I.Y. Arefeva, P.B. Medvedev and A.P. Zubarev, New representation for string field solves the consistency problem for open superstring field theory, Nucl. Phys. B341 (1990), 464-498.

[8] N. Berkovits, Super-Poincaré invariant superstring field theory, Nucl. Phys. B450 (1995), 90-102, [arXiv:hep-th/9503099].

[9] N. Berkovits, A. Sen and B. Zwiebach, Tachyon condensation in superstring field theory, Nucl. Phys. B587 (2000), 147-178, [arXiv:hep-th/0002211].

[10] N. Berkovits, The Ramond sector of open superstring field theory, J. High Energy Phys. 11 (2001), 47, [arXiv:hep-th/0109100].

[11] I.Y. Arefeva, D.M. Belov and A.A. Giryavets, Construction of the vacuum string field theory on a non-BPS brane, J. High Energy Phys. 9 (2002), 50, [arXiv:hep-th/0201197]. 
[12] Y. Michishita, A covariant action with a constraint and Feynman rules for fermions in open superstring field theory, J. High Energy Phys. 1 (2005), 12, [arXiv:hep-th/0412215].

[13] N. Berkovits, Pure spinor formalism as an $N=2$ topological string, J. High Energy Phys. 10 (2005), 89, [arXiv:hep-th/0509120].

[14] N. Berkovits and W. Siegel, Regularizing cubic open NeveuSchwarz string field theory, J. High Energy Phys. 11 (2009), 21, [arXiv:0901.3386].

[15] M. Kroyter, On string fields and superstring field theories, J. High Energy Phys. 8 (2009), 44, [arXiv:0905.1170].

[16] M. Kroyter, Superstring field theory equivalence: Ramond sector, J. High Energy Phys. 10 (2009), 44, [arXiv:0905.1168].

[17] M. Kroyter, Comments on superstring field theory and its vacuum solution, J. High Energy Phys. 8 (2009), 48, [arXiv:0905.3501].

[18] E. Fuchs and M. Kroyter, Analytical solutions of open string field theory, Phys. Rep. 502 (2011), 89-149.

[19] P.-J. De Smet and J. Raeymaekers, The tachyon potential in Witten's superstring field theory, J. High Energy Phys. 8 (2000), 20, [arXiv: hep-th/0004112].

[20] N. Berkovits, Relating the RNS and pure spinor formalisms for the superstring, J. High Energy Phys. 8 (2001), 26, [arXiv:hep-th/ 0104247].

[21] P.A. Grassi and M. Schnabl, unpublished.

[22] T. Erler, Tachyon vacuum in cubic superstring field theory, J. High Energy Phys. 1 (2008), 13, [arXiv:0707.4591].

[23] I.Y. Aref'eva, R.V. Gorbachev and P.B. Medvedev, Tachyon solution in cubic Neveu-Schwarz string field theory, Theor. Math. Phys. 158 (2009), 320-332, [arXiv:0804.2017].

[24] E. Fuchs and M. Kroyter, On the classical equivalence of superstring field theories, J. High Energy Phys. 10 (2008), 54, [arXiv:0805. 4386].

[25] N. Berkovits and C. Vafa, N=4 topological strings, Nucl. Phys. B433 (1995), 123-180, [arXiv:hep-th/9407190].

[26] M. Schnabl, Analytic solution for tachyon condensation in open string field theory, Adv. Theor. Math. Phys. 10 (2006), 433-501, [arXiv:hep-th/0511286].

[27] I. Ellwood and M. Schnabl, Proof of vanishing cohomology at the tachyon vacuum, J. High Energy Phys. 2 (2007), 96, [arXiv:hep-th/0606142]. 
[28] I. Ellwood, B. Feng, Y.-H. He and N. Moeller, The identity string field and the tachyon vacuum, J. High Energy Phys. 7 (2001), 16, [arXiv: hep-th/0105024].

[29] F.J. Narganes-Quijano, Picture changing operation and BRST cohomology in superstring field theory, Phys. Lett. B212 (1988), 292.

[30] J.N. Acosta, N. Berkovits and O. Chandia, A note on the superstring BRST operator, Phys. Lett. B454 (1999), 247-248, [arXiv:hep-th/9902178].

[31] C.G. Callan, Jr., C. Lovelace, C.R. Nappi and S.A. Yost, Loop corrections to superstring equations of motion, Nucl. Phys. B308 (1988), 221.

[32] M. Kiermaier, Y. Okawa and B. Zwiebach, The boundary state from open string fields, [arXiv:0810.1737].

[33] G.T. Horowitz, R.C. Myers and S.P. Martin, BRST cohomology of the superstring at arbitrary ghost number, Phys. Lett. B218 (1989), 309.

[34] B.H. Lian and G.J. Zuckerman, BRST cohomology of the supervirasoro algebras, Commun. Math. Phys. 125 (1989), 301.

[35] N. Berkovits, Quantization of the superstring with manifest $U(5)$ super-poincaré invariance, Phys. Lett. B457 (1999), 94-100, [arXiv:hep-th/9902099].

[36] J. Zinn-Justin, Renormalization of gauge theories. Lectures given at Int. Summer Inst. for Theoretical Physics, Bonn, West Germany, July 29-August 9, 1974.

[37] I.A. Batalin and G.A. Vilkovisky, Gauge algebra and quantization, Phys. Lett. B102 (1981), 27-31.

[38] I.A. Batalin and G.A. Vilkovisky, Quantization of gauge theories with linearly dependent generators, Phys. Rev. D28 (1983), 2567-2582.

[39] I.A. Batalin and G.A. Vilkovisky, Closure of the gauge algebra, generalized Lie equations and Feynman rules, Nucl. Phys. B234 (1984), 106-124.

[40] B.L. Voronov and I.V. Tyutin, Formulation of gauge theories of general form I, Theor. Math. Phys. 50 (1982), 218-225.

[41] M. Henneaux, Lectures on the antifield-BRST formalism for gauge theories, Nucl. Phys. Proc. Suppl. 18A (1990), 47-106.

[42] M. Henneaux and C. Teitelboim, Quantization of gauge systems, University Press, Princeton, USA, 1992, 520pp.

[43] J. Gomis, J. Paris and S. Samuel, Antibracket, antifields and gauge theory quantization, Phys. Rep. 259 (1995), 1-145, [arXiv:hep-th/9412228]. 
[44] C.B. Thorn, Perturbation theory for quantized string fields, Nucl. Phys. B287 (1987), 61.

[45] M. Bochicchio, String field theory in the Siegel gauge, Phys. Lett. B188 (1987), 330.

[46] M. Bochicchio, Gauge fixing for the field theory of the bosonic string, Phys. Lett. B193 (1987), 31.

[47] C.B. Thorn, String field theory, Phys. Rep. 175 (1989), 1-101.

[48] E. D'Hoker and D.H. Phong, Lectures on two-loop superstrings, [arXiv:hep-th/0211111].

[49] A. Morozov, NSR superstring measures revisited, J. High Energy Phys. 5 (2008), 86, [arXiv:0804.3167].

[50] P. Dunin-Barkowski, A. Morozov and A. Sleptsov, Lattice theta constants vs Riemann theta constants and NSR superstring measures, J. High Energy Phys. 10 (2009), 72, [arXiv:0908.2113].

[51] M. Kiermaier, A. Sen and B. Zwiebach, Linear b-gauges for open string fields, J. High Energy Phys. 3 (2008), 50, [arXiv:0712.0627].

[52] M. Asano and M. Kato, New covariant gauges in string field theory, Prog. Theor. Phys. 117 (2007), 569-587, [arXiv:hep-th/ 0611189].

[53] M. Asano and M. Kato, General linear gauges and amplitudes in open string field theory, Nucl. Phys. B807 (2009), 348-372, [arXiv:0807.5010].

[54] M. Kroyter, Democratic superstring field theory: Gauge fixing, J. High Energy Phys. 03 (2011), 81.

[55] N. Berkovits, A new description of the superstring, [arXiv:hep-th/ 9604123].

[56] Y. Okawa, Comments on Schnabl's analytic solution for tachyon condensation in Witten's open string field theory, J. High Energy Phys. 4 (2006), 55, [arXiv:hep-th/0603159].

[57] E. Fuchs, M. Kroyter and R. Potting, Marginal deformations in string field theory, J. High Energy Phys. 9 (2007), 101, [arXiv:0704. 2222 [hep-th]].

[58] E. Fuchs and M. Kroyter, Marginal deformation for the photon in superstring field theory, J. High Energy Phys. 11 (2007), 5, [arXiv:0706.0717 [hep-th]].

[59] Z.-A. Qiu and A. Strominger, Gauge symmetries in (super)string field theory, Phys. Rev. D36 (1987), 1794.

[60] N. Berkovits, Super-Poincare covariant quantization of the superstring, J. High Energy Phys. 4 (2000), 18, [arXiv:hep-th/0001035]. 
[61] N. Berkovits, ICTP lectures on covariant quantization of the superstring, [arXiv:hep-th/0209059].

[62] Y. Aisaka, E.A. Arroyo, N. Berkovits and N. Nekrasov, Pure spinor partition function and the massive superstring spectrum, J. High Energy Phys. 8 (2008), 50, [arXiv:0806.0584].

[63] O.A. Bedoya and N. Berkovits, GGI lectures on the pure spinor formalism of the superstring, [arXiv:0910.2254].

[64] N. Berkovits, Explaining the pure spinor formalism for the superstring, J. High Energy Phys. 1 (2008), 65, [arXiv:0712.0324].

[65] N. Berkovits and B. Zwiebach, On the picture dependence of Ramond-Ramond cohomology, Nucl. Phys. B523 (1998), 311-343, [arXiv:hep-th/9711087].

[66] R. Saroja and A. Sen, Picture changing operators in closed fermionic string field theory, Phys. Lett. B286 (1992), 256-264, [arXiv: hep-th/9202087].

[67] N. Berkovits, Y. Okawa and B. Zwiebach, WZW-like action for heterotic string field theory, J. High Energy Phys. 11 (2004), 38, [arXiv:hep-th/0409018].

[68] A. Sen, Descent relations among bosonic D-branes, Int. J. Mod. Phys. A14 (1999), 4061-4078, [arXiv:hep-th/9902105].

[69] A. Sen, Universality of the tachyon potential, J. High Energy Phys. 12 (1999), 27, [arXiv:hep-th/9911116].

[70] E. Fuchs and M. Kroyter, On the validity of the solution of string field theory, J. High Energy Phys. 5 (2006), 6, [arXiv:hep-th/0603195]. 
